\title{
Co-occurrence of transcription and translation gene regulatory features underlies coordinated mRNA and protein synthesis
}

\author{
Ana Tamarkin-Ben-Harush ${ }^{1}$, Edna Schechtman ${ }^{2}$ and Rivka Dikstein ${ }^{*}$
}

\begin{abstract}
Background: Variability in protein levels is generated through intricate control of the different gene decoding phases. Presently little is known about the links between the various gene expression stages. Here we investigated the relationship between transcription and translation regulatory properties encoded in mammalian genes.

Results: We found that the TATA-box, a core promoter element known to enhance transcriptional output, is associated not only with higher mRNA levels but also with positive translation regulatory features and elevated translation efficiency. Further investigation revealed general association between transcription and translation regulatory trends. Specifically, translation inhibitory features such as the presence of upstream AUG (UAUG) and increased lengths of the $5^{\prime}$ UTR, the coding sequence and the $3^{\prime}$ UTR, are strongly associated with lower translation as well as lower transcriptional rate.

Conclusions: Our findings reveal that co-occurrence of several gene-encoded transcription and translation regulatory features with the same trend substantially contributes to the final mRNA and protein expression levels and enables their coordination.
\end{abstract}

Keywords: TATA-box, TATA-less, Transcription, Translation, UAUG, 5'UTR, 3'UTR, uORF

\section{Background}

Expression of protein-encoding gene in eukaryotes is an intricate process that includes several distinct steps of transcription, mRNA processing and mRNA translation. Each of these stages is controlled by cis-regulatory elements present in the DNA and the mRNA. Transcription is governed by two major types of DNA elements, enhancer and core promoter. Enhancer elements serve as binding sites for transcription regulatory factors and can function independently of their position. Core promoter elements, such as TATA-box and Initiator, are situated around the transcription start site (TSS) and are the sites on which the basal transcription machinery assembles. As such these elements have central role in determining promoter strength [1-3].

Cis-regulatory elements present in the mRNA are central to the control of protein synthesis. Specifically the

\footnotetext{
* Correspondence: rivka.dikstein@weizmann.ac.il

'Department of Biological Chemistry, The Weizmann Institute of Science, Rehovot 76100, Israel

Full list of author information is available at the end of the article
}

nucleotide sequence surrounding the initiating AUG $[4,5]$, the presence of an AUG(s) upstream to the main ORF (uAUGs) $[4,6-8]$, the lengths of the $5^{\prime}$ and $3^{\prime}$ untranslated regions (UTRs) and the occurrence of stemloop structures in the $5^{\prime}$ UTR [9-14], all influence the rate of protein synthesis. Previous genomic and functional studies suggest that uAUGs act to reduce translation of the downstream ORF either of specific genes or globally $[4,6-8]$. The presence of uAUG in eukaryotic mRNAs is highly prevalent, reaching almost half of protein coding genes $[6,15]$.

Here we investigated the relationship between various regulatory features of transcription and translation encoded in mammalian genes using bioinformatics and functional analyses. Our findings revealed remarkable coupling of several regulatory features that act in the same direction which substantially contribute to mRNA and protein levels and facilitate their coordinated expression. 


\section{Results}

The highly transcribed TATA-box genes have lower frequency of uAUG

The TATA box is a well-characterized strong core promoter element that is known to be associated with high transcriptional rate [16-21]. Previously we have shown that TATA-containing genes tend to have short length and reduced 5' and 3'UTR size [20]. However the relationships of these and other features such as uAUGs and coding sequence (CDS) length with translation efficiency were not investigated. To address these issues we identified the TATA-box genes by searching the -40 to -15 region, relative to the annotated TSS of the UCSC database, for the TATAWAG sequence (allowing zero to one mismatch). With this definition of the TATA-box the frequency of this motif is $5 \%$ and $8.5 \%$ in human and mouse genes, respectively. The same frequency of the TATA-box was found with the DBTSS database, which contains TSSs from CAGE (Cap Analysis of Gene Expression) data. We then compared the frequency of uAUG in all genes to genes containing or lacking TATAbox in their promoter. Consistent with earlier reports [22-24] we found that a considerable fraction of human $(\sim 47 \%)$ and rodent ( 40\%) mRNAs possess at least one uAUG in their 5'UTR (Figure 1A and B). Interestingly, in both human and mouse the percentage of uAUG bearing genes is the lowest in the canonical TATA-box group, higher in the one-mismatch TATA-box and the highest in TATA-less group (Figure $1 \mathrm{~A}$ and $\mathrm{B}$ ). In other words the frequency of the TATA-box among uAUG genes is lower than uAUG-less genes (3.9\% vs. 5.7\% in human and $6 \%$ vs. $10.2 \%$ in mouse, respectively). Thus the prevalence of uAUG negatively correlates with the presence and the strength of the TATA-box. We also carried gene ontology analysis of the uAUG and uAUG-less genes and found some differences with enrichment of several functional categories (Additional file 1: Table S1).
TATA-box genes lacking uAUG are associated with positive translation regulatory features and higher translation

To examine further the relationship between various translation regulatory features human and mouse genes were first grouped as either lacking or containing uAUG (uAUG-less and uAUG, respectively). As shown in Figure 2, remarkable differences exist between the two gene sets both in human and in mouse. The 5'UTR of uAUG-less genes is substantially shorter than that of uAUG genes (Figure 2A). This pattern was repeated with the 3'UTR length and the ORF (CDS) length: uAUG-less genes tend to have significantly shorter 3'UTR and ORF than uAUG genes (Figure 2B and C). While the length of the $5^{\prime} \mathrm{UTR}$ may be linked to the presence of uAUG, the lengths of the 3'UTR and the CDS have no apparent natural connection to the presence or absence of uAUG in the 5'UTR, yet these translation regulatory traits tend to cluster on mRNAs.

Next we compared the translation regulatory features between the TATA (with up to one mismatch) and the TATA-less groups, each divided into uAUG and uAUGless subsets. We found dramatic differences in all parameters among the uAUG-less subsets, in both human and mouse (Figure 3). Specifically, the 5'UTR, the 3'UTR and the ORF lengths were significantly shorter in the TATA than in TATA-less genes. However in the uAUG containing genes the differences between TATA and TATA-less are much smaller (Additional file 1: Figure S1). These findings are consistent with those reported previously [20] but the present analysis revealed that these differences exist primarily among the uAUG-less subsets. Thus the TATAbox genes that lack uAUG are associated with additional positive translation regulatory features.

To test the relationship between regulatory features of genes and protein synthesis we retrieved genome-wide translation efficiency data from two recent ribosomeprofiling studies from mouse cells. The first contained data of 4,840 genes from mouse embryonic fibroblasts
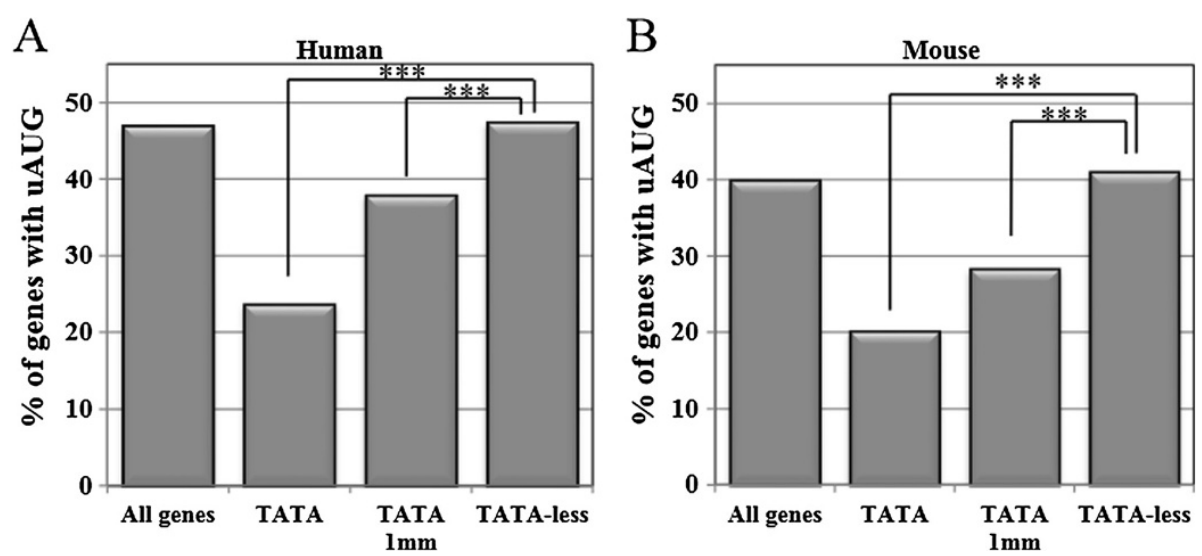

Figure 1 The prevalence of UAUG in human (A) and mouse (B) genes bearing canonical TATA, TATA with up to one mismatch (1 mm) or TATA-less. All genes refer to the sum of TATA (with up to one mismatch) and TATA-less genes. ${ }^{* *}$ denotes statistically significant difference $p<0.001$. 


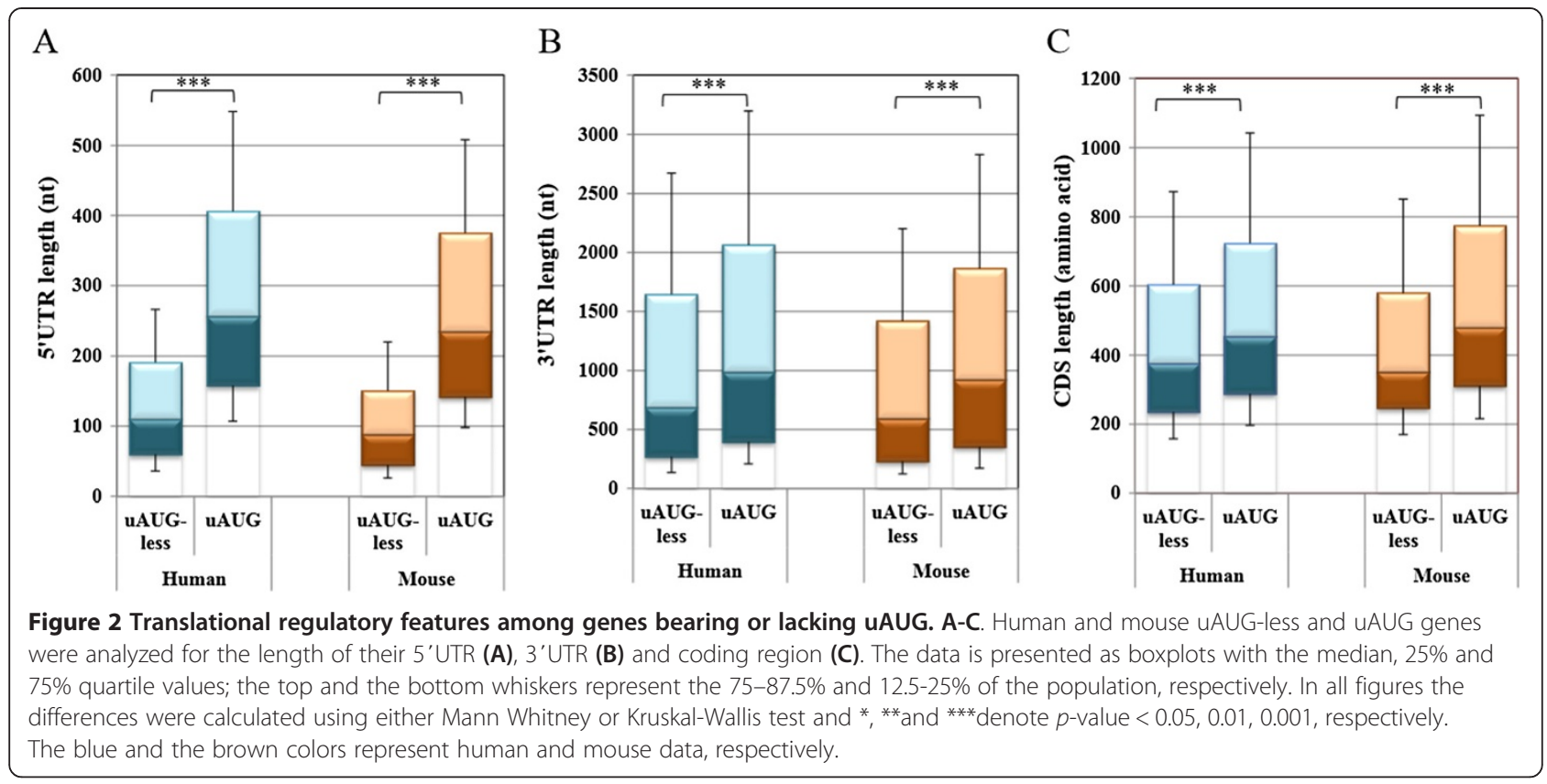

(MEFs) [25] and the second of 10,220 genes from mouse embryonic stem cells (mESCs) [26]. The relationship between 5'UTR, 3'UTRs and the CDS lengths with translation efficiency was assessed using a Spearman rank correlation coefficient. The results revealed a moderate but significant negative correlation between 5'UTR $(-0.226, \quad \mathrm{p}<0.0001)$ and 3'UTR $(-0.429, \quad \mathrm{p}<0.0001)$ lengths and translation efficiency ( $\mathrm{TE}=$ ribosome reads/ total mRNA). The negative correlation between ORF length and translation efficiency was very small $(-0.058$, $\mathrm{p}<0.0001)$ and may be explained by the RNA-seq methodology used in these studies. For the analysis of the translational activity we calculated the ribosomal density of each gene, which is the ratio between the TE of each transcript and the length of the coding sequence (TE/CDS length). Assessment of ribosomal density of uAUG-less and uAUG in MEFs and in mESCs revealed that the uAUG-less genes show significantly greater ribosomal density than uAUG genes (Figure 4A), which is in agreement with the notion that uAUG attenuates translation from the major ORF.

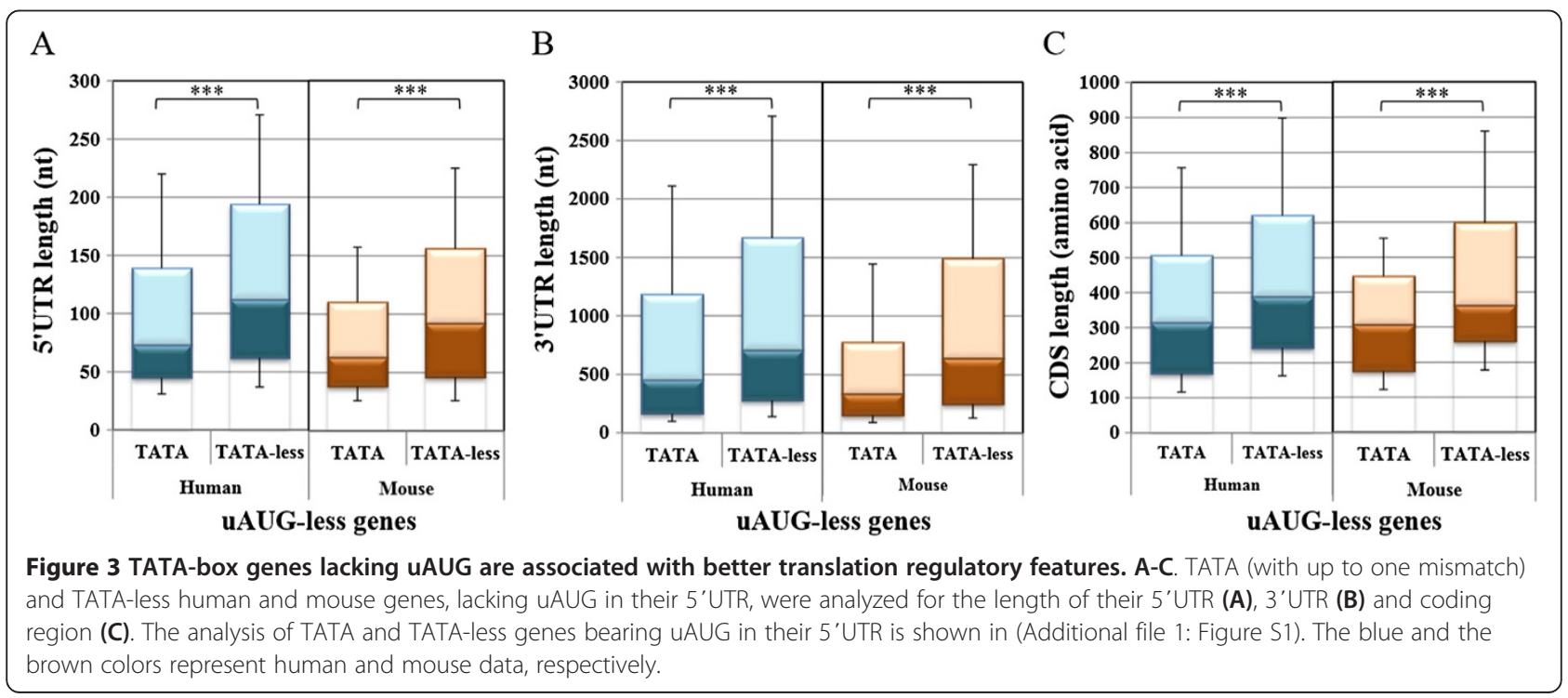




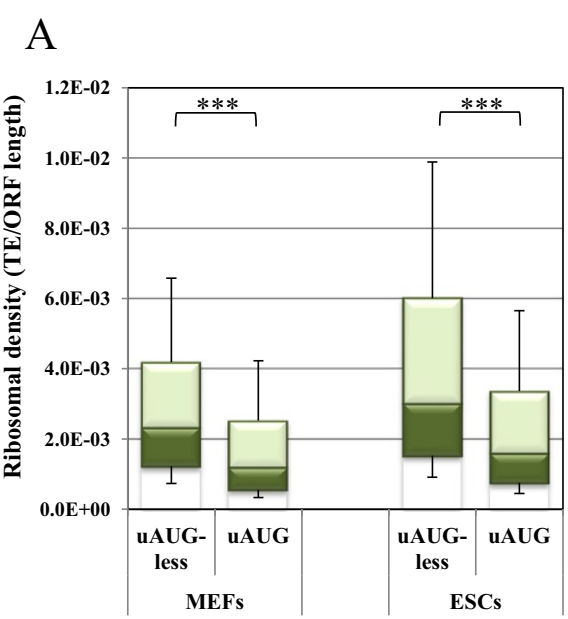

C

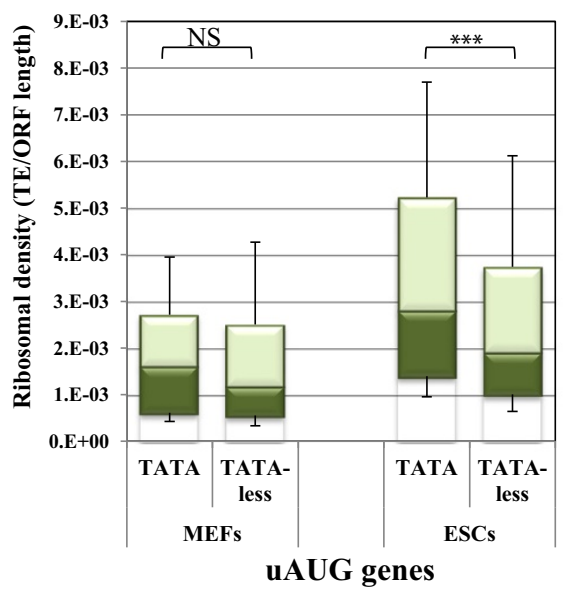

B

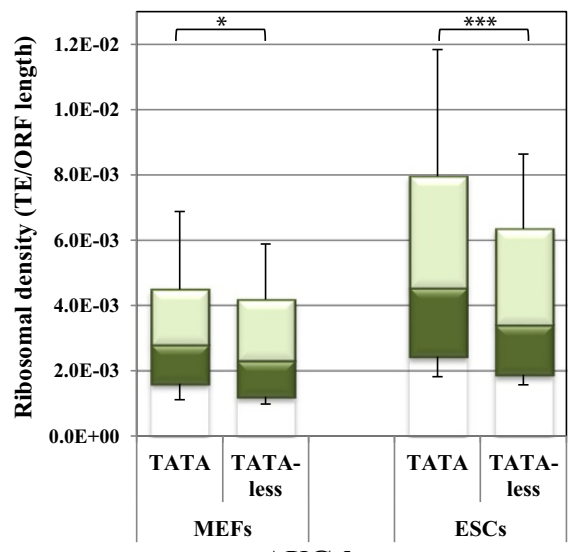

uAUG-less genes

Figure 4 TATA-box genes are associated with higher translational activity. A. The translational activity of mouse genes lacking (UAUG-less) or bearing UAUG (UAUG) is presented as ribosomal density values which were derived from two global ribosomal profiling experiments in MEFs and mESC $[25,26]$ and presented as boxplots. B. Ribosomal density values of TATA (with up to one mismatch) and TATA-less mouse uAUG-less genes from the ribosomal profiling data described above. C. Ribosomal density values of TATA and TATA-less mouse genes bearing uAUG.

The green color of the bar represent the data taken from mouse ribosomal profiling studies $[25,26]$.

Considering that TATA genes are associated with positive translational features we would expect these highly transcribed genes to be efficiently translated. Therefore the two datasets were used to compare the translation levels of TATA and TATA-less genes, containing or lacking uAUG. We observed that among the uAUG-less genes, the TATA set showed significantly higher ribosomal density levels than that of the TATA-less set both in MEFs and mESCs (Figure 4B). While with the MEFs no significant differences were seen between the UAUG genes, with the ESCs the ribosomal density of the TATA set was higher (Figure 4C). Together, the analysis of the regulatory features and translational activities support the notion that regulatory traits in transcription and translation were evolved to act in a similar trend.

\section{Co-occurrence of translation and transcription regulatory trends}

As a positive transcription regulatory element such as TATA-box was found to be associated with positive translation regulatory features we were prompted to examine general links between transcription and translation. We first analyzed the relationship between ribosomal density and the mRNA levels by Spearman rank correlation coefficient analysis, using the data retrieved from the ribosomal profiling experiment described above $[25,26]$. Interestingly, significant positive correlation of $0.418(\mathrm{p}<0.0001)$ was found between ribosomal density and mRNA levels. As ribosomal density, represents the efficiency by which each mRNA molecule is translated, independently of the number of RNA molecules, this correlation is unexpected and 
is not the same as the correlation between mRNA abundance and protein abundance reported previously $[27,28]$. To gain further insight into the underlying basis of this connection we compared the transcript levels between the uAUG-less and uAUG gene sets. Remarkably, uAUG-less genes, which are translated more efficiently, tend to have significantly higher mRNA reads in both MEFs and mESCs measurements (Figure 5A). Likewise we found negative correlations between $\mathrm{mRNA}$ levels and translation features such as 5'UTR $(-0.2, \mathrm{p}<0.0001)$, ORF length $(-0.461, \mathrm{p}<0.0001)$ and 3 'UTR length $(-0.368$, $\mathrm{p}<0.0001)$.

To examine further the relationship between mRNA levels and translational features observed for mouse genes, we similarly analyzed human gene expression data that was downloaded from the gene expression atlas SymAtlas v1.2.3. This database contains expression data of thousands of human genes from 79 tissues and cell types. We determined the average expression of each gene in all tissues, setting a threshold of 200 , a value that is above background. Then we determined the distribution of the average expression of each gene in uAUG-less and uAUG sets using boxplots. Here again it appears that human uAUG-less genes tend to have significantly higher levels of mRNA than uAUG genes (Figure $5 \mathrm{~B}$ ). This is particularly highlighted in the upper $50 \%$ of the gene population that is distributed more towards the higher expression levels, both in the human and the mouse data (Figure 5A and B).

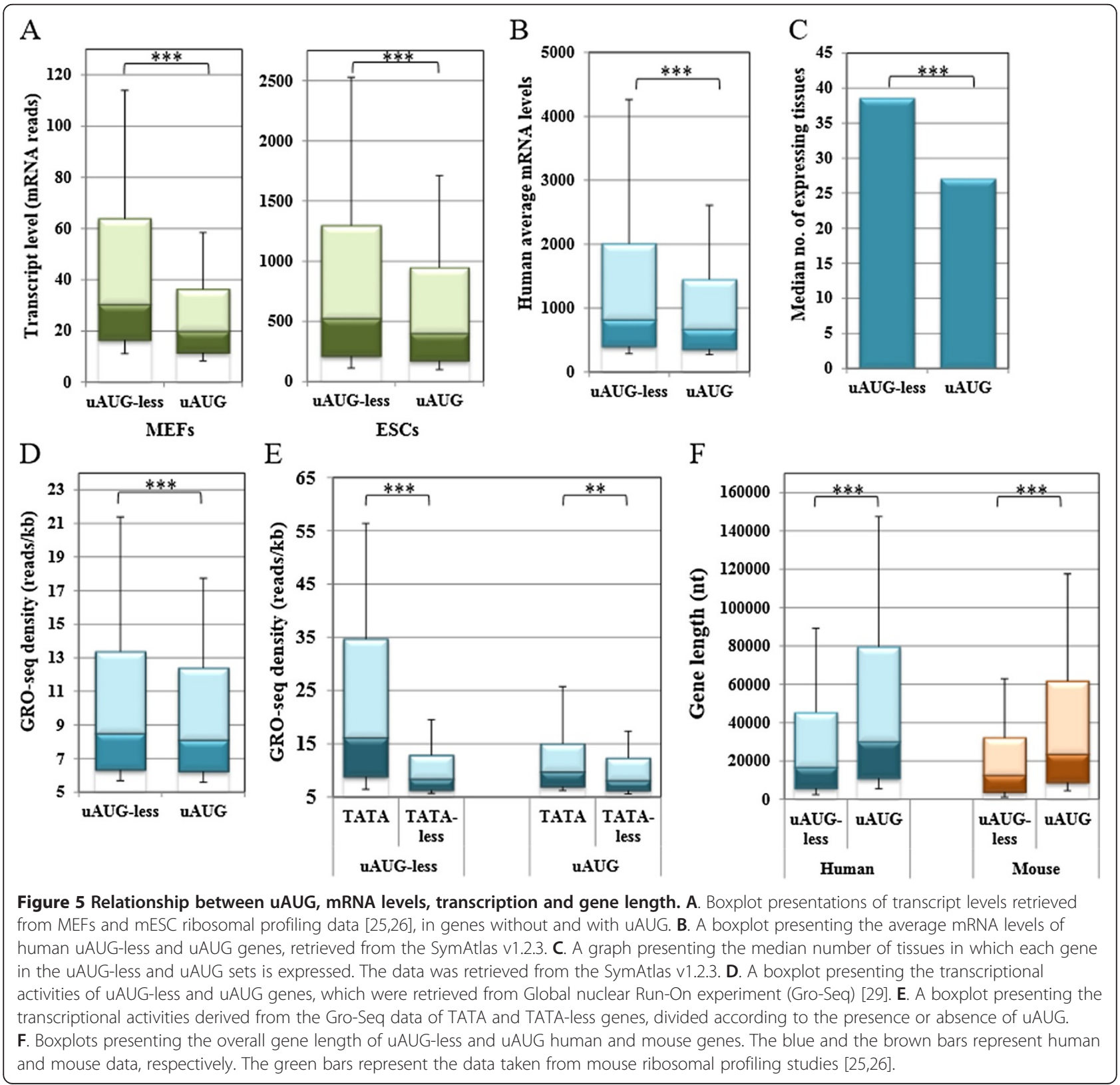


A similar pattern is observed when the maximal, rather than the average expression of genes is analyzed (Additional file 1: Figure S2). The number of tissues in which each gene is expressed was also determined in the two gene sets, and we found that uAUG-less genes tend to be expressed in more tissues than uAUG genes (Figure $5 \mathrm{C}$ ).

The analysis shown in Figure 5A and $\mathrm{B}$ is derived from steady state mRNA levels data. To examine whether the transcription process directly contributes to the differences seen in mRNA levels between uAUG-less and uAUG gene we retrieved RNA-seq data from a global Nuclear Run-On experiment (Gro-seq), which directly measures the level of ongoing transcription for all genes [29]. The same general trend was found, as uAUG-less genes display higher levels of transcription than those with uAUG (Figure 5D). These findings support the idea that uAUG genes are less efficiently transcribed than uAUG-less genes. It has been previously suggested that $\mathrm{uAUG}$ is associated with a shorter mRNA half-life [23], therefore it can be presumed that the combined effects of lower transcription and elevated decay rates are responsible for the marked difference in the steady state mRNA levels (Figure 5A and B). We also analyzed the transcription efficiency of TATA and TATA-less genes divided into uAUG-less and uAUG subsets. The results revealed the expected differences between TATA and TATA-less genes but remarkably this difference is much less dramatic among the uAUG genes (Figure 5E).

An important parameter that is known to influence transcription efficiency is the gene length $[20,30]$. Upon analysis we found substantial differences in gene length between uAUG-less and uAUG genes in human and in mouse (Figure 5F), the median gene length in uAUG-less genes being almost half of that in uAUG genes. Exon count analysis showed the same trend (Additional file 1: Figure S3).

Next, expression data from multiple tissues of 6804 human genes were divided with into top $25 \%$ and bottom $75 \%$ expressed genes and determined the percentage of uAUG bearing genes in the two groups and found that the top $25 \%$ gene set has lower uAUG genes than the bottom 75\% set (Additional file 1: Figure S4A). The prevalence of uAUG in the top 10\% expressed genes is even lower. A similar trend was observed with the GROseq data (data not shown). To examine whether it is just the presence of the UAUG that is associated with the reduced mRNA levels we compared the translational regulatory features of the top $25 \%$ and bottom $75 \%$ expressed genes (at the mRNA level) within the same class, either uAUG-less or uAUG (Additional file 1: Figure S4B-E). While no significant difference is observed in the $5^{\prime}$ UTR length between the high and the low expressing genes, clear and marked differences are seen with the lengths of 3'UTR, CDS and overall gene size, these features being much shorter in the higher expressing set both in uAUG- less and uAUG groups. These findings clearly show that while uAUG and the 5'UTR length are important, they are insufficient to account for the association between translational and transcriptional features reinforcing that the co-occurrence of other features also contribute to final expression levels.

\section{Discussion and conclusions}

The present study demonstrates that various transcription and translation regulatory features were co-evolved in the same direction. Specifically we observed that translation regulatory features acting positively or negatively are linked to transcriptional control features, such as TATAbox and gene length, that function in the corresponding direction. Our findings suggest that clustering of various structural as well as regulatory features, which have the same trend but at different stages of gene expression, can be regarded as a powerful and general mechanism for coordinating the various gene expression stages. This coordination is particularly apparent in the TATA-box gene set as illustrated in Figure 6. In transcription, the TATA-box acts by increasing the rate of initiation $[19,31,32]$. TATAbox genes are also very short and have fewer introns [20] therefore their transcription elongation and mRNA processing is more efficient. The combined effects of these features give rise to high levels of mRNAs [20]. On the translation side, the TATA-box gene set is also characterized by shorter $5^{\prime}$ and $3^{\prime}$ UTRs, smaller ORF size and

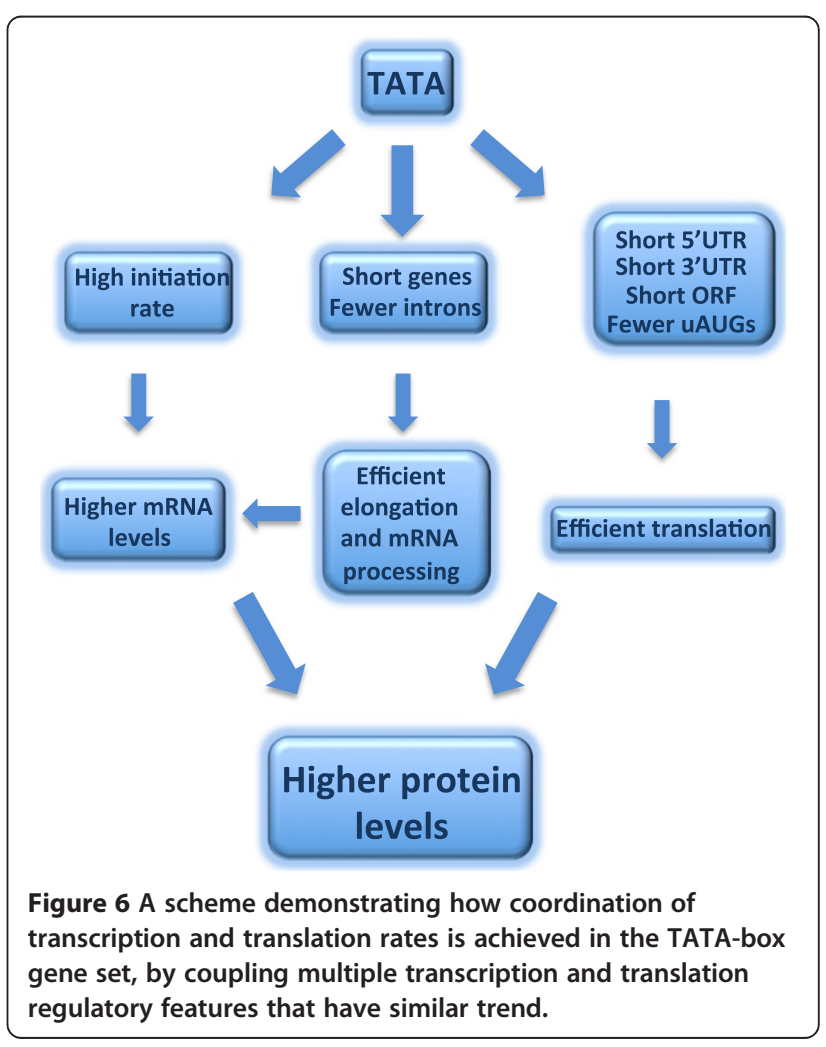


lower incidence of uAUG. Consistent with that we show that the mRNA molecules generated from these genes tend to be more efficiently translated. Thus it appears that the coupling of all these transcription and translation regulatory features results in much higher level of protein production. An exception is the fraction of TATA genes that also have uAUG. In these genes the advantage of the TATA box in transcription seem to be lost and is accompanied with high prevalence of translation inhibitory features and lower translation.

The link between transcriptional and translational features is not limited to the TATA gene class as analysis of structural organization and functional features of mammalian genes bearing or lacking uAUG, revealed close association with mRNA levels and transcriptional rate as well as other translation regulatory traits that display the same trend. The negative correlation between mRNA abundance and uAUG has been previously noted [23,27] but this was mostly attributed to the reduction in steady-state mRNA levels and mRNA half-life. The analysis of the Gro-Seq data which reflects only mRNA synthesis rate, revealed that the lower mRNA level associated with uAUG is a combination of lower synthesis with lower stability.

Several recent studies uncovered the associations between features present in the mRNA and protein abundance in yeast and mammalian cells (for example see $[27,28,33])$. These studies also reported that mRNA features, such as lengths of the $5^{\prime}$ UTR, $3^{\prime}$ UTR and CDS as well as UORF are associated with each other as we also find here. Our analysis further extends these findings by demonstrating that the translational activity of each mRNA is correlated with mRNA abundance. Furthermore, we show that 5'UTR, 3'UTR and CDS as well as $\mathrm{uORF}$ are also associated with genomic features that influence the rate of mRNA synthesis, in particular promoter features (TATA vs. TATA-less) and gene length.

A major biological implication resulting from our findings is the ability of the eukaryotic cell to synchronize, to some extent, the transcription and translation rates, through various regulatory features operating in the same direction. The gene ontology analysis (Additional file 1: Table S1) which revealed enrichment of functional categories associated with uAUG and uAUG-less genes, provides examples for coordination of expression level with biological activity. For instance, transcription factors are known to be transcribed at low levels and indeed these factors have higher prevalence of uAUG. On the other hand structural components such as nucleosomal proteins that are highly expressed at the mRNA and protein levels tend to lack uAUG.

In summary, the analysis of transcription and translation data reported here revealed significant association between mRNA levels that reflect transcriptional activity and decay, and translation efficiency.

\section{Methods}

\section{Selection of genes and analyzing their features}

Gene data and sequences were retrieved from UCSC Genome Browser website (http://genome.ucsc.edu/) in which the Feb. 2009 assembly was used for human genes and July 2007 assembly for mouse genes. Using RefSeq track in 'Table browser' we downloaded the desired genomic sequence output (CDS and UTRs) for the different groups of genes. To identify uAUG-bearing genes the $5^{\prime}$ UTR sequence of all genes were retrieved and analyzed by a PERL code designed to identify the ATG triplet. Genes were subsequently divided into two groups: with and without AUG triplet codon in their 5'UTR (uAUG and uAUG-less, respectively). Sequences of the gene set of interest were analyzed in Galaxy (https://usegalaxy. org/root), a web-based platform for data managing [34-36] and, using EMBOSS 'infoseq' tool the length of the sequences was retrieved. Gene length was calculated using the difference between transcription start and end positions in 'selected fields' output format. The number of exons was retrieved from exonCount field. Ribosomal density was determined as follows:

$$
\text { Ribosomal density }=\frac{\frac{\text { ribosome reads }}{\text { total mRNA reads for each transcript }}}{\text { CDS length }}
$$

Classification of genes according to their function was done using the gene-annotation enrichment analysis (http://david.abcc.ncifcrf.gov/).

\section{Identification of TATA-box bearing genes}

The nucleotides sequences from -40 to -15 upstream to the UCSC TSS, were retrieved. Using the 'pattern matching' tool in Regulatory Sequence Analysis Tools (RSAT) site (http://rsat.ulb.ac.be/) we searched for the TATA-box sequence of TATAWAG (allowing zero to one mismatch) in this region. The RefSeq output was transformed into official gene name to remove duplicates.

\section{Gene expression}

Gene expression data was analyzed as previously described [20]. The transcription Gro-Seq data was retrieved from Core et al., [29]. To avoid bias generated by proximal promoter pausing the reads of the first $1 \mathrm{~kb}$ were avoided. The number of reads divided by the gene length (minus $1 \mathrm{~kb}$ ), reflected the transcription level of each gene. A ratio below 5 reads/kb was considered background. For mouse mRNA levels the total RNA-seq data derived from the ribosomal profiling studies $[25,26]$ were used.

\section{Statistical analyses of gene features}

The distributions of the gene features are skewed therefore non-parametric procedures were used to compare between the groups features, Mann-Whitney U-test for 
two and Kruskal-Wallis test for more than two samples. The differences between uAUG prevalence among the groups was analyzed by chi-square test and the Spearman's rank correlation coefficient analysis between MEF's translation efficiencies and the length of mRNA features was performed using the STATSTICA ${ }^{\circ}$ software.

\section{Additional file}

Additional file 1: Figure S1. Translational regulatory features among TATA and TATA-less genes bearing UAUG. A-C. Human and mouse genes containing UAUG in their 5'UTR were grouped according to the presence and absence of a TATA-box in their core promoter region and analyzed for the length of their 5'UTR (A), 3'UTR (B) and coding region (C). The data is presented as boxplots with the median, $25 \%$ and $75 \%$ quartile values; the top and the bottom whiskers represent the $75-87.5 \%$ and $12.5-25 \%$ of the population, respectively. In all figures the differences were calculated using the Kruskal-Wallis test and * and ${ }^{* * *}$ denote $p$-value $<0.05$ and 0.001 , respectively. NS, statistically non significant. The blue and the brown bars represent human and mouse data, respectively. Figure S2: A boxplot presenting the maximal mRNA levels of human uORF-less and uORF genes, retrieved from the SymAtlas v1.2.3. Figure S3: Boxplots presenting the number of exons in human and mouse uORF-less and UORF genes. The blue and the brown bars represent human and mouse data, respectively. Figure S4: Highly mRNA expressing genes are associated with better translational features. A. The prevalence of uAUG in the top 10\%, 25\% and the bottom 75\% mRNA expressing genes. The differences are statistically significant $p<10^{-4}$. B-E. Top $25 \%$ and bottom $75 \%$ mRNA expressing genes, containing or lacking UAUG were analyzed for the length of their 5'UTR (B), 3'UTR (C), coding region (D) and gene length (E). Table S1: Enrichment of functional categories of uAUG-less and uAUG genes.

\section{Abbreviations}

CAGE: Cap Analysis of Gene Expression; CDS: Coding sequence; Gro-seq: Global Nuclear Run-On experiment; MEFs: Mouse embryonic fibroblasts; mESCs: Mouse embryonic stem cells; RSAT: Regulatory Sequence Analysis Tools; TE: Translation efficiency; TSS: Transcription start site; UAUG: Upstream AUG; UTR: Un-translated region.

\section{Competing interests}

The authors declare no competing financial and non-financial interests.

\section{Authors' contributions}

ATBH conceived and designed the study, performed the bioinformatics and statistical analyses of the various gene features and wrote this paper. ES participated in the statistical analysis of the data. RD conceived and designed the study, analyzed the data and wrote the paper. All authors read and approved the final manuscript.

\section{Acknowledgements}

We are grateful to Eliezer Dikstein for his assistance in programming, Dr. Sandra Moshonov for critical reading and editing of the manuscript, Tali Shalit (INCPM Unit, WIS) for processing the Gro-seq data, Nicholas T. Ingolia (UCSF) and Carson C. Thoreen (Whitehead Institute for Biomedical Research) for providing the RNA-seq data of the ribosomal profiling. This work was supported by grants from the Minerva Foundation (\#711124) and the Israel Science Foundation (\#1168/13). R.D. is the incumbent of the Ruth and Leonard Simon Chair of Cancer Research.

\section{Author details}

${ }^{1}$ Department of Biological Chemistry, The Weizmann Institute of Science, Rehovot 76100, Israel. ${ }^{2}$ Department of Industrial Engineering and Management, Ben Gurion University of the Negev, Beer Sheva 84105, Israel.

Received: 10 April 2014 Accepted: 14 August 2014

Published: 19 August 2014

\section{References}

1. Dikstein R: The unexpected traits associated with core promoter elements. Transcription 2011, 2(5):201-206.

2. Juven-Gershon T, Hsu JY, Kadonaga JT: Perspectives on the RNA polymerase II core promoter. Biochem Soc Trans 2006, 34(Pt 6):1047-1050.

3. Smale ST, Kadonaga JT: The RNA polymerase II core promoter. Annu Rev Biochem 2003, 72:449-479.

4. Kozak M: Selection of initiation sites by eucaryotic ribosomes: effect of inserting AUG triplets upstream from the coding sequence for preproinsulin. Nucleic Acids Res 1984, 12(9):3873-3893.

5. Kozak M: Initiation of translation in prokaryotes and eukaryotes. Gene 1999, 234(2):187-208.

6. Calvo SE, Pagliarini DJ, Mootha VK: Upstream open reading frames cause widespread reduction of protein expression and are polymorphic among humans. Proc Natl Acad Sci U S A 2009, 106(18):7507-7512.

7. Meijer HA, Thomas AA: Control of eukaryotic protein synthesis by upstream open reading frames in the $5^{\prime}$-untranslated region of an mRNA. Biochem J 2002, 367(Pt 1):1-11.

8. Morris DR, Geballe AP: Upstream open reading frames as regulators of mRNA translation. Mol Cell Biol 2000, 20(23):8635-8642.

9. Kozak M: Influences of mRNA secondary structure on initiation by eukaryotic ribosomes. Proc Natl Acad Sci U S A 1986, 83(9):2850-2854.

10. Kozak M: Structural features in eukaryotic mRNAs that modulate the initiation of translation. The J Biological chemistry 1991, 266(30):19867-19870

11. Kozak M: A short leader sequence impairs the fidelity of initiation by eukaryotic ribosomes. Gene Expr 1991, 1(2):111-115.

12. Kozak M: Effects of long 5 ' leader sequences on initiation by eukaryotic ribosomes in vitro. Gene Expr 1991, 1(2):117-125.

13. Kuersten S, Goodwin EB: The power of the 3' UTR: translational control and development. Nat Rev Genet 2003, 4(8):626-637.

14. Mazumder B, Seshadri V, Fox PL: Translational control by the 3'-UTR: the ends specify the means. Trends Biochem Sci 2003, 28(2):91-98.

15. Wethmar K, Barbosa-Silva A, Andrade-Navarro MA, Leutz A: uORFdb-a comprehensive literature database on eukaryotic uORF biology. Nucleic Acids Res 2014, 42:60-67.

16. Amir-Zilberstein L, Ainbinder E, Toube L, Yamaguchi Y, Handa H, Dikstein R: Differential regulation of NF-kappaB by elongation factors is determined by core promoter type. Mol Cell Biol 2007, 27(14):5246-5259.

17. Blake WJ, Balazsi G, Kohanski MA, Isaacs FJ, Murphy KF, Kuang Y, Cantor CR, Walt DR, Collins JJ: Phenotypic consequences of promoter-mediated transcriptional noise. Mol Cell 2006, 24(6):853-865.

18. Hoopes BC, LeBlanc JF, Hawley DK: Contributions of the TATA box sequence to rate-limiting steps in transcription initiation by RNA polymerase II. J Mol Biol 1998, 277(5):1015-1031.

19. Marbach-Bar N, Ben-Noon A, Ashkenazi S, Harush AT, Avnit-Sagi T, Walker MD Dikstein R: Disparity between microRNA levels and promoter strength is associated with initiation rate and Pol II pausing. Nat Commun 2013, 4:2118

20. Moshonov S, Elfakess R, Golan-Mashiach M, Sinvani H, Dikstein R: Links between core promoter and basic gene features influence gene expression. BMC Genomics 2008, 9(1):92.

21. Wobbe CR, Struhl $K$ : Yeast and human TATA-binding proteins have nearly identical DNA sequence requirements for transcription in vitro. Mol Cell Biol 1990, 10(8):3859-3867

22. lacono M, Mignone F, Pesole G: UAUG and uORFs in human and rodent 5' untranslated mRNAs. Gene 2005, 349:97-105.

23. Matsui M, Yachie N, Okada Y, Saito R, Tomita M: Bioinformatic analysis of post-transcriptional regulation by $\mathrm{UORF}$ in human and mouse. FEBS Lett 2007, 581(22):4184-4188.

24. Mignone F, Gissi C, Liuni S, Pesole G: Untranslated regions of mRNAs. Genome Biol 2002, 3(3):REVIEWS0004.

25. Thoreen CC, Chantranupong L, Keys HR, Wang T, Gray NS, Sabatini DM: A unifying model for mTORC1-mediated regulation of mRNA translation. Nature 2012, 485(7396):109-113.

26. Ingolia NT, Lareau LF, Weissman JS: Ribosome profiling of mouse embryonic stem cells reveals the complexity and dynamics of mammalian proteomes. Cell 2011, 147(4):789-802.

27. Vogel C, Abreu Rde S, Ko D, Le SY, Shapiro BA, Burns SC, Sandhu D, Boutz DR, Marcotte EM, Penalva LO: Sequence signatures and mRNA concentration can explain two-thirds of protein abundance variation in a human cell line. Mol Syst Biol 2010, 6:400. 
28. Schwanhausser B, Busse D, Li N, Dittmar G, Schuchhardt J, Wolf J, Chen W, Selbach M: Global quantification of mammalian gene expression control. Nature 2011, 473(7347):337-342.

29. Core LJ, Waterfall JJ, Lis JT: Nascent RNA sequencing reveals widespread pausing and divergent initiation at human promoters. Science 2008, 322(5909):1845-1848.

30. Swinburne IA, Miguez DG, Landgraf D, Silver PA: Intron length increases oscillatory periods of gene expression in animal cells. Genes Dev 2008, 22(17):2342-2346.

31. Morachis JM, Murawsky CM, Emerson BM: Regulation of the $\mathrm{p} 53$ transcriptional response by structurally diverse core promoters. Genes Dev 2010, 24(2):135-147.

32. Yean D, Gralla J: Transcription reinitiation rate: a special role for the TATA box. Mol Cell Biol 1997, 17(7):3809-3816.

33. Zur $\mathrm{H}$, Tuller $\mathrm{T}$ : Transcript features alone enable accurate prediction and understanding of gene expression in S. cerevisiae. BMC Bioinformatics 2013, 14(Suppl 15):S1.

34. Blankenberg D, Von Kuster G, Coraor N, Ananda G, Lazarus R, Mangan M, Nekrutenko A, Taylor J: Galaxy: a web-based genome analysis tool for experimentalists. Curr Protoc Mol Biol/ edited by Frederick M Ausubel [et al] 2010, Chapter 19(Unit 19 10):11-21.

35. Goecks J, Nekrutenko A, Taylor J, Galaxy T: Galaxy: a comprehensive approach for supporting accessible, reproducible, and transparent computational research in the life sciences. Genome Biol 2010, 11(8):R86.

36. Giardine B, Riemer C, Hardison RC, Burhans R, Elnitski L, Shah P, Zhang Y, Blankenberg D, Albert I, Taylor J, Miller W, Kent WJ, Nekrutenko A: Galaxy: a platform for interactive large-scale genome analysis. Genome Res 2005, 15(10):1451-1455.

doi:10.1186/1471-2164-15-688

Cite this article as: Tamarkin-Ben-Harush et al: Co-occurrence of transcription and translation gene regulatory features underlies coordinated mRNA and protein synthesis. BMC Genomics 2014 15:688.

\section{Submit your next manuscript to BioMed Central and take full advantage of:}

- Convenient online submission

- Thorough peer review

- No space constraints or color figure charges

- Immediate publication on acceptance

- Inclusion in PubMed, CAS, Scopus and Google Scholar

- Research which is freely available for redistribution 